\title{
Assessing Left Ventricular Ejection Time from Wrist Cuff Pulse Waveforms:
}

\author{
Algorithm and Evaluation
}

\author{
Jiri Jilek \\ Carditech \\ Culver City \\ California \\ USA \\ jilekj@usa.net
}

\author{
Milan Stork \\ Department of Applied Electronics and \\ Telecommunications/RICE \\ University of West Bohemia \\ Plzen, Czech Republic \\ stork@kae.zcu.cz
}

\begin{abstract}
Left ventricular ejection time (LVET) is an important component of systolic time intervals. It has been used as a surrogate for left ventricular contractility and stroke volume. We developed a method for the assessment of LVET from wrist cuff pulse waveforms. The waveforms are obtained from a partially inflated cuff held at the level of the heart. Each test involved acquisition of 10 second sample of waveform data. The data were processed after each test by specially developed software algorithm. Thirty tests were evaluated and compared with predicted values. The results showed the mean value of computed LVET equal to $309 \mathrm{~ms}$ and mean value of predicted LVET equal to $304 \mathrm{~ms}$. We concluded that our method has the potential for use as a stand-alone method for evaluation of left ventricular contractility and for the determination of stroke volume.
\end{abstract}

Keywords- systolic time intervals (STI), left ventricular ejection time (LVET), wrist cuff pulse waveforms, BlandAltman plot, stroke volume (SV)

\section{INTRODUCTION}

Systolic time intervals (STI) reflect cardiac performance [1]. Left ventricular ejection time (LVET) is an essential component of STI. LVET is the time interval of the left ventricular ejection which starts with the opening of aortic valve and ends with its subsequent closure. LVET is related to left ventricular contractility and to stroke volume (SV). Shortened LVET indicates increased contractility and prolonged LVET is directly proportional to SV. Stroke volume can be used to calculate cardiac output [2] and to calculate systemic arterial compliance. Current standard method for noninvasive determination of LVET is echocardiography. Both M-mode and doppler ultrasound methods have been used. Echocardiographic methods require, however, expensive instrumentation and special operator skills. Less expensive methods not requiring special skills have been explored. Phonocardiography, impedance cardiography, seismocardiography, and

Milan Stork's participation was supported by Department of Applied Electronics and Telecommunications, University of West Bohemia, Plzen and by the Ministry of Education, Youth and Sports of the Czech Republic under the RICE and New Technologies and Concepts for Smart Industrial Systems, project No. LO1607 and by the Internal Grant Agency of University of West Bohemia in Pilsen, Czech Republic the project SGS-2015-002 and GA15-22712S. photoplethysmography have gained a measure of importance. The authors of a recent study [3] compared several noninvasive methods and came to the conclusion that echocardiography has less variability than phonocardiography or photoplethysmography.

LVET has been used for estimation of stroke volume. Impedance cardiography uses LVET and the rate of impedance change for each given beat to compute SV [4]. Impedance cardiography has been used commercially.

We previously developed a versatile dual-cuff experimental system for determination of blood pressure (BP) and hemodynamics [5]. The system incorporates an arm cuff and a wrist cuff. The wrist cuff can be used independently for the assessment of wrist BP and hemodynamics. Figure 1 shows a volunteer in sitting position with wrist cuff on the left wrist.

In this study we used the wrist cuff pulse waveforms for the determination of LVET. We described the global algorithm that processes the waveforms and determines the LVET. Results from 30 tests were compared with predicted LVET values.

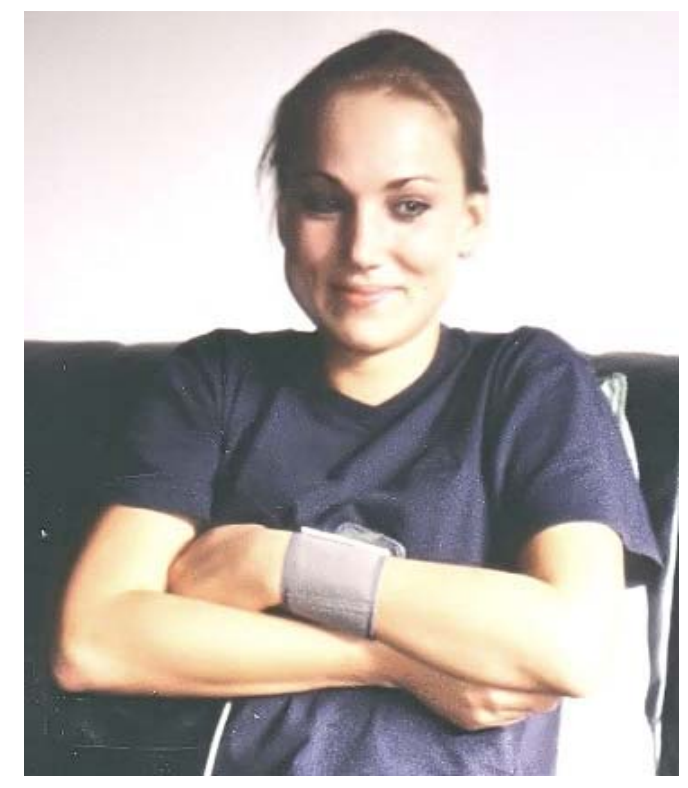

Figure 1. Wrist cuff on the left wrist of a sitting volunteer. 


\section{METHODS}

The wrist cuff waveforms and wrist cuff pressures were acquired and stored in a database. The cuff pressures were not used for this study because they were not needed for determination of LVET. Cuff pressure was monitored for the determination of preinflation of the wrist cuff. The waveforms were used for the development of an algorithm that was considered an improvement on the previous algorithm.

\section{A. Instrumentation}

The dual cuff system contains two sub-systems that can be used independently. The arm cuff subsystem is not used in this study. The wrist cuff subsystem (Figure 2) consists of pneumatic and electronic circuits. The pneumatic circuit consists of a $6 \mathrm{~cm}$ wide wrist cuff, an air pump and a valve that are controlled by a sub-module. The analog circuit uses a piezoelectric pressure transducer, amplifier and a filter. The amplified and filtered analog signal is digitized by a 12-bit ADC. We used 85 samples/sec data rate. The digital data is sent to a notebook computer via USB connection. The notebook computer uses Windows-based specially developed software which features several functions. We used the function "Wrist cuff test". This test inflates the wrist cuff to a sub- diastolic cuff pressure and holds this pressure for 10 seconds while cuff pressure and pulse waveforms are acquired. At the end of the $10 \mathrm{sec}$ segment the notebook performs the required computations. The raw data is then stored for future use.

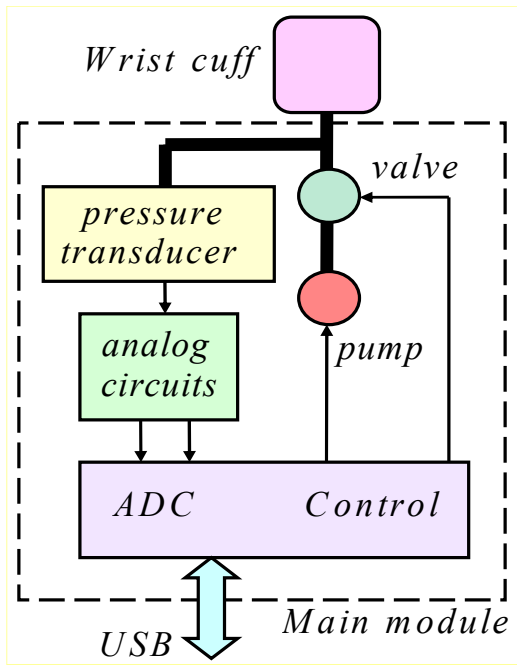

Figure 2. Block diagram of the wrist cuff sub-system consists of pneumatic and electronic circuits.

\section{B. Rationale for our algorithm for LVET determination}

The systolic cycle of the left ventricle can be observed on the wrist cuff pulse waveforms (Figure 3). The beginning of systolic upstroke corresponds with the opening of the aortic valve. Late systole corresponds with the downturn of the waveform contour. The end of systole is marked by closure of aortic valve. This point on the waveform is called dicrotic wave or notch. The interval from the notch to the onset of the next interval corresponds to the duration of diastole. The LVET corresponds to time interval measured from the onset of the waveform to the dicrotic notch. The waveform onset and the dicrotic notch are frequently difficult to determine accurately, especially in older individuals.

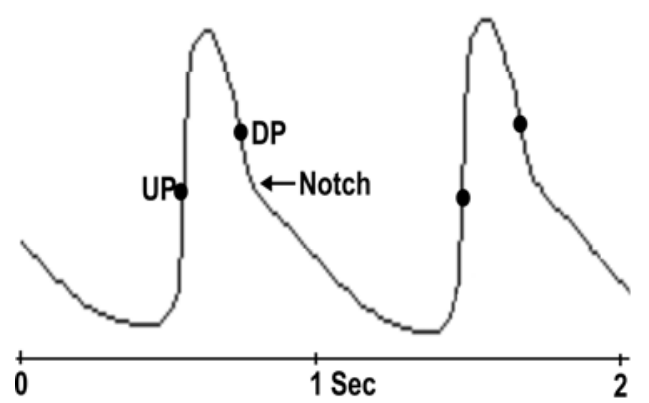

Figure 3. Wrist cuff pulse waveforms used for determination. UP is upstroke point, DP is the downstroke point and Notch is the dicrotic notch.

Our research into an optimal LVET algorithm showed that the most accurate and consistent way to find these two points on the waveform is to find the largest amplitude increment per one sample interval on the upslope (UP) and downslope (DP) points. Because the UP and DP points do not correspond to the onset and the notch, time adjustment must be applied.

Determination of heart rate (HR) is also necessary because we used predicted LVET values $\left(\right.$ LVET $_{\mathrm{P}}$ ) developed by Weissler [6] for comparison with the wrist cuff method. Weissler found close relationship between resting values of LVET and HR in healthy individuals. The LVET values in Weissler's study were derived noninvasively from the carotid arterial pulse. The carotid artery method is similar to the wrist cuff method because it measures LVET as the interval from the onset to the dicrotic notch of the carotid pulse waveform. The formula below determines this relationship:

$$
\operatorname{LVET}_{\mathrm{P}}=-1.65 \cdot \mathrm{HR}+415 \quad\left[\mathrm{~ms}, \text { beat.sec }{ }^{-1}\right]
$$

\section{Algorithm for LVET and HR determination}

The algorithm describes global steps of the sequence of the software routines. Detailed description of those routines is beyond the scope of this paper. The systolic upstroke point UP is the position of the largest amplitude increment per one sample interval $(11.8 \mathrm{msec})$. The late systolic downstroke point DP is the position of the largest amplitude increment of the late systole

1. Determine time position of the systolic upstroke point (UP in Figure 3))

2. Adjust point position for onset and store adjusted UP point position.

3. Determine time position of late systolic downstroke point (DP in Figure 3).

4. Adjust for notch point position and store adjusted DP point position.

5. Compute $\mathrm{LVET}_{\mathrm{M}}$ (DP - UP) and store LVET $_{M}$. 
6. Move to next waveform, repeat steps $1-5$ until all waveforms are done.

7. Compute and store mean $\mathrm{LVET}_{\mathrm{M}}$

8. Compute HR from time intervals between successive waveforms.

9. Compute and store predicted $\operatorname{LVET}_{\mathrm{P}}$ value from formula (1).

Steps 2 and 4 involve software routines that adjust the UP and DP positions variably depending on the waveform slopes. Steeper slopes required smaller adjustment value than the less steep slopes.

The above algorithm was used to compute values in 30 tests performed on healthy volunteers ( 26 males and 4 females) in sitting position and resting. The $\mathrm{LVET}_{\mathrm{M}}$ values for each test are mean values computed from $10 \mathrm{sec}$ periods.

\section{RESULTS}

The results from 30 tests were stored in a Microsoft Excel file. Excel was then used to calculate mean values and standard deviations of $\mathrm{LVET}_{\mathrm{M}}$, $\mathrm{LVET}_{\mathrm{P}}$ and HR. The results are shown in Table 1.

TABLE I. MEAN VALUES AND STANDARD DEVIATIONS (SD) FOR LVET AND HR. LVET VALUES ARE IN MILLISECONDS (MS). HR VALUES ARE IN BEAT PER MINUTE.

\begin{tabular}{|c|c|c|c|}
\hline N=30 & LVET $_{\mathbf{M}}$ & LVET $_{\mathbf{P}}$ & HR \\
\hline MEAN & 309 & 304 & 66 \\
\hline SD & 19.8 & 18.4 & 10.7 \\
\hline
\end{tabular}

The mean values of $\mathrm{LVET}_{\mathrm{M}}$ and $\mathrm{LVET}_{\mathrm{P}}$ are quite close; the difference is only $5 \mathrm{~ms}$ or about $1.6 \%$. The mean values do not, however, show differences between values obtained from individual tests.

\section{DISCUSSION}

Because the wrist cuff method of LVET determination is novel, we decided to show how the $\mathrm{LVET}_{\mathrm{M}}$ values differ from the predicted values of $\mathrm{LVET}_{\mathrm{P}}$ developed by Weissler [6]. Bland and Altman developed a method [7] of data plotting used in analyzing the agreement between two different methods. We used this plot to show agreement between $\operatorname{LVET}_{\mathrm{M}}$ and $\mathrm{LVET}_{\mathrm{P}}$. This approach is valid because both $\mathrm{LVET}_{\mathrm{M}}$ and $\mathrm{LVET}_{\mathrm{P}}$ values are derived from the same raw data sets. Bland-Altman plot in Figure 4 shows the differences between measured $\mathrm{LVET}_{\mathrm{M}}$ and predicted $\mathrm{LVET}_{\mathrm{P}}$ for 30 values computed from the tests. The plot shows good agreement between the measured and the predicted LVET values. Ninety percent of the differences were within the 2SD limits. There was a small positive bias of $5 \mathrm{~ms}$. The more extreme LVET values corresponding to low and high HR values fell within the 2SD limits. The mean value of $\mathrm{LVET}_{\mathrm{M}}$ and $\mathrm{LVET}_{\mathrm{P}}$ of $245 \mathrm{~ms}$ corresponded to $\mathrm{HR}$ value of $96 \mathrm{bpm}$. The mean value of $\mathrm{LVET}_{\mathrm{M}}$ and $\mathrm{LVET}_{\mathrm{P}}$ of $345 \mathrm{~ms}$ corresponded to $\mathrm{HR}$ value equal to $44 \mathrm{bpm}$. The 30 test data sets were obtained from volunteers in sitting posture and resting. In different postures, in response to exercise, and in certain cardiovascular diseases the predicted values based on HR lose some of their validity because they are based on data obtained from normal subjects in sitting position and resting.

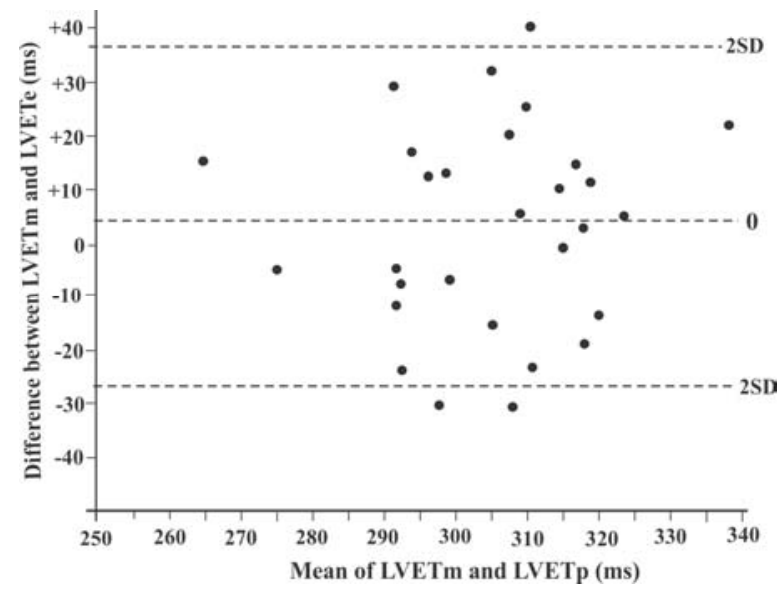

Figure 4. Bland-Altman plot of agreement between LVET $_{M}$ and LVET$_{\mathrm{P}}$. The horizontal axis shows the mean values of $\mathrm{LVET}_{\mathrm{M}}$ and $\mathrm{LVET}_{\mathrm{P}}$. The vertical axis shows the differences between $\mathrm{LVET}_{M}$ and $\operatorname{LVET}_{\mathrm{P}}$

To illustrate this point we used a test performed immediately after bicycle exercise. The measured $\mathrm{LVET}_{\mathrm{M}}$ was $309 \mathrm{~ms}$ and the $\mathrm{LVET}_{\mathrm{P}}$ predicted from heart rate $(\mathrm{HR}=101 \mathrm{BPM})$ was $242 \mathrm{~ms}$. The postexercise $\mathrm{LVET}_{\mathrm{M}}$ interval was $67 \mathrm{~ms}$ longer than $\mathrm{LVET}_{\mathrm{P}}$ predicted from HR. The prolongation of LVET is related to increased stroke volume in exercise [8]. The LVET predicted from resting HR cannot reflect this change. On the other hand, the test in our study with $\mathrm{HR}=96 \mathrm{bpm}$ resting value showed good agreement between $\mathrm{LVET}_{\mathrm{P}}$ and $\mathrm{LVET}_{\mathrm{M}}$. Prolongation or shortening of LVET due to certain cardiovascular diseases cannot be determined from predicted LVET. A study of 40 patients with aortic valvular stenosis [9] found LVET significantly prolonged when compared with heart rate derived predicted values. The $\mathrm{LVET}_{\mathrm{M}}$ determined with the wrist cuff method may be able to detect this prolongation.

In addition to utilization of LVET as a measure of left ventricular contractility, LVET is an important variable used for the determination of stroke volume (SV) and cardiac output (CO) in impedance cardiography [4]. We previously used LVET for the determination of SV from wrist cuff pulse waveforms [10]. The algorithm developed for this study is an improved version that should contribute to improved accuracy of SV and $\mathrm{CO}$ determination. Additionally, LVET is used indirectly for the determination of total peripheral resistance (TPR) and systemic arterial compliance (SAC). TPR is determined from mean arterial pressure and cardiac output. SAC is calculated from pulse pressure and stroke volume. We included the determination of TPR and SAC in our previous study [10].

\section{CONCLUSION}

We concluded that the wrist cuff method could be used as a stand-alone method for evaluation of left ventricular function and for the determination of 
stroke volume. In this study we used the comparison with LVET values derived from resting heart rate values. Next step in the development will be comparison of the wrist cuff method with ultrasound method which is considered to be the gold standard for noninvasive determination of systolic time intervals. It would also be beneficial to assess the wrist cuff method used on sick patients.

\section{REFERENCES}

[1] P. Reamt, M. Dijos, E. Donal, A. Mignot et al, "Systolic time intervals as simple echocardiographic parameters of left ventricular performance: correlation with ejection fraction and longitudinal two-dimensional strain," European Journal of Echocardiography 2010, pp. 834-844.

[2] R. M. Berne, M. N. Levy, "Cardiovascular Physiology, " Mosby 1992, pp. 144-146.

[3] W.Duan, D. Zheng, C. Eggett, P. Langley, A. Murray, "Development of techniques for measurement of left ventricular ejection time," Computing in Cardiology 2014; 41, pp. 241-244.
[4] H. H. Voltjer, H. J. Bogaard, J. M. deVries, "The technique of impedance cardiography," European Heart Journal 1997; 18:, pp.1396-1403.

[5] J. Jilek, M. Stork, "Dual cuff system improves noninvasive blood pressure determination," Applied Electronics, AE2010 International Conference proceedings, Plzen, Czech Republic: 2010, pp. 125-129.

[6] A. M. Weissler, W. S. Harris, C. D. Schoenfeld, "Systolic time intervals in heart failure in man," Circulation, No: 21, 1968, pp. 149-155.

[7] J. M. Bland, D. G. Altman, "Statistical methods for assessing agreement between two methods of clinical measurement, " The Lancet 1986, pp. 307-311.

[8] D. R. Lamb, "Physiology of exercise," MacMillan 1984, pp. 144-146.

[9] R. J. Bache, Y. Wang, J. C. Greenfield, "Left Ventricular Ejection Time in Valvular Aortic Stenosis," Circulation 1973, 47; pp 527-533.

[10] J. Jilek, M. Stork, "The use of radial oscillometric waveforms in estimation of blood pressure and hemodynamics," WSEAS, International Conference, Rhodes, Greece, 2009. 